\title{
Stochastic dynamics of Ginzburg-Landau vortices in superconductors
}

\author{
Jennifer Deang* \\ Department of Mathematics, Virginia Tech, Blacksburg, Virginia 24060-0123 \\ Qiang Du and Max D. Gunzburger \\ Department of Mathematics, Iowa State University, Ames, Iowa 50011-2064
}

(Received 6 September 2000; revised manuscript received 19 January 2001; published 13 July 2001)

\begin{abstract}
Thermal fluctuations and randomly distributed defects in superconductors are modeled by stochastic variants of the time-dependent Ginzburg-Landau equations. Numerical simulations are used to compare the effects of additive and multiplicative noise models.

DOI: 10.1103/PhysRevB.64.052506

PACS number(s): 74.20.De, 74.40.+k, 74.60.Ge, 74.80.- $-\mathrm{g}$
\end{abstract}

\section{INTRODUCTION}

The phenomenological Ginzburg-Landau model for lowtemperature superconductivity has received much attention. However, it is not applicable to physical contexts that do not take into account factors such as material defects or thermal fluctuations. Thus, existing studies of the stability, dynamics, interactions, and other properties of the vortex state do not necessarily carry over to situations for which these factors cannot be ignored.

The effects of defects and thermal fluctuations play a central role in the motion and pinning of vortices in type-II superconductors. ${ }^{1}$ In this Brief Report, we examine the vortex dynamics in type-II superconductors based on stochastic versions of the Ginzburg-Landau model. For simplicity, we assume that the underlying material sample possesses a large value for the Ginzburg-Landau parameter $\kappa$ so that a reduction of the Ginzburg-Landau equations to their high- $\kappa$ limit can be employed. ${ }^{2,3}$ In this case, the given applied field penetrates the sample completely. We will consider different forms for the stochastic perturbations of the high- $\kappa$ limit Ginzburg-Landau model and illustrate, through numerical experiments, their effect on the dynamics of vortices.

\section{STOCHASTIC HIGH- $\kappa$ GINZBURG-LANDAU MODEL}

Although at each point in the material and at each instant in time the fluctuations or defects have negligible effect, the superposition of many interactions can produce observable effects. The fluctuations or defects, being random in magnitude and direction, produce instantaneous changes in the movement of vortices. In Ref. 1, it is argued that thermodynamic fluctuations are generally quite small, which corresponds to a small value for the variance. We consider two approaches of adding random fluctuations or defects to a high- $\kappa$ Ginzburg-Landau model: the cases of additive and linear multiplicative noise. The comparison of the two cases in other physically meaningful systems has received much attention; see, e.g., Ref. 4.

Let $\Omega \in \mathbb{R}^{d}$ denote the superconducting sample and $\Gamma$ its boundary. The derivation of the high- $\kappa$ model in a deterministic setting starts with the time-dependent Ginzburg-Landau (G-L) equations. ${ }^{5}$ Then, as the G-L parameter $\kappa \rightarrow \infty$ (with the gauge choice of the electric potential $\Phi$ being zero in $\Omega$ and the magnetic potential $\mathbf{A}$ having zero normal component on $\Gamma$ ), the resulting simplified leading-order system is a nonlinear equation for the leading-order (in terms of $1 / \kappa^{2}$ ) order parameter $\psi$, after ignoring the effects of applied currents and assuming that the applied field is of order $\kappa: 2,3$

$$
\frac{\partial \psi}{\partial t}+(i \nabla+\mathbf{A})^{2} \psi-\psi+|\psi|^{2} \psi=0 \quad \text { in } \Omega,
$$

along with the boundary condition $\nabla \psi \cdot \mathbf{n}=0$ on $\Gamma$ and the initial condition $\psi(\mathbf{x}, 0)=\varphi(\mathbf{x})$ in $\Omega$. The magentic potential A, a deterministic vector field, is solved for separately from the given applied field $\mathbf{H}_{0}$ using Maxwell's equations.

Additive noise high- $\kappa$ model. With $\eta$ being a random, complex-valued field in time and space, the additive noise high- $\kappa$ G-L model is of Langevin type and is given by ${ }^{6-12}$

$$
\frac{\partial \psi}{\partial t}+(i \nabla+\mathbf{A})^{2} \psi-\psi+|\psi|^{2} \psi=\eta \quad \text { in } \Omega .
$$

The random source term $\eta$ is assumed (see Ref. 8) to be noise generated by an infinite dimensional Brownian motion. ${ }^{13}$ If the effects of thermal fluctuations are being modeled, the variance $\sigma$ of $\eta$ is temperature dependent, i.e., $\sigma=K\left(T / T_{c}\right)\left(1-T / T_{c}\right)^{-2}\left(T\right.$ and $T_{c}$ denote the temperature and the critical transition temperature, respectively) for some temperature independent constant $K$. Note that $\sigma \rightarrow \infty$ as $T$ $\rightarrow T_{c}$ so that the effects of thermal fluctuations should be large near $T_{c}$.

We note that in Eqs. (1) and (2) the space variables have been nondimensionalized with respect to the temperature dependent coherence length $\xi(T)=\xi(0)\left(1-T / T_{c}\right)^{-1 / 2}$. In our computational simulations, we will vary the variance of the noise or, equivalently, we will vary the temperature. As a result, because of our nondimensionalization scale, changing the variance implies that we are also changing $\xi$. This means that as we change the value of variance the spatial scale used for nondimensionalization changes. The relation between $\xi$ and $\sigma$ is easily found to be $[\xi(T) / \xi(0)]^{2}=(1$ $+\sqrt{1+4 \sigma / K}) / 2$ so that for large values of $\sigma$ we have that $\xi(T) \propto \sigma^{1 / 4}$. Thus, for example, doubling the variance in our computational simulations means that, for large variances, we are enlarging the linear dimension of the material sample 

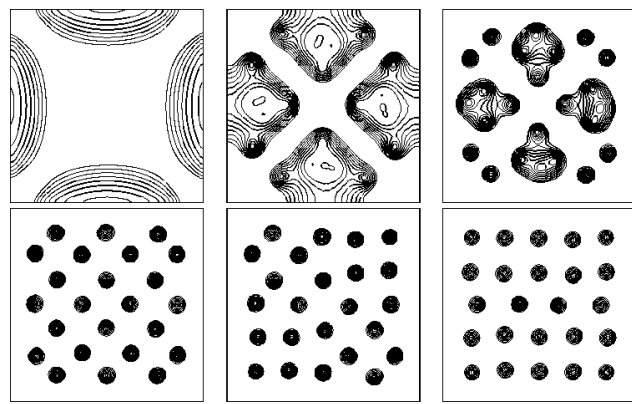

FIG. 1. Time evolution of $|\psi|$ in a $20 \xi \times 20 \xi$ homogeneous, isotropic sample with no thermal fluctuations; the bottom right figure is the steady state configuration.

by a factor of 1.19. It should be noted that the scale used for nondimensionalizing the time is also temperature dependent.

Multiplicative noise high- $\kappa$ model. A multiplicative noise high- $\kappa$ G-L model is given by replacing Eq. (1) by

$$
\frac{\partial \psi}{\partial t}+(i \nabla+\mathbf{A})^{2} \psi-\psi+|\psi|^{2} \psi=\eta \psi \quad \text { in } \Omega .
$$

In Eq. (3), the noise term $\eta$ appears as a coefficient of a term that is linear in $\psi$; one could more generally introduce other nonlinear stochastic terms. It is not clear if multiplicative noise can model the effect of thermal fluctuations and if the models satisfy the fluctuation-dissipation theorem; see Ref. 8. Indeed, one of our goals is to see what is the effect of different noise models on the dynamics of vortices.

However, multiplicative noise of the type used in Eq. (3) has been used to model defects in superconductors; see, e.g., Ref. 14. In this case, the nature of the noise depends on the scale of the inhomogeneities. If these are smaller than a G-L coherence length $\xi$, one may assume that $\eta$ is independent in space and time. On the other hand, if the inhomegeneities are correlated over distances comparable to a coherence length, then the coefficient $\eta$ should have a similar correlation length scale. See Ref. 14.

Discretization of noise. To carry out the computational simulations, we must discretize the random functions. For the additive noise model, we assume that $\eta$ is independent in space and time. Thus, we approximate $\eta$ by a grid function whose nodal values at all grid points and time steps are sampled as independent random variables with a Gaussian distribution of zero mean and variance $\sigma$. Consequently, if one refines the grid used for the solution of Eq. (2) one also refines the sampling points for the approximation of $\eta$.

In the multiplicative noise case, we have experimented with discretized noise similar to that used in the additive noise case, corresponding to noise that is correlated over length scales smaller than the coherence length. We have also experimented with discretized noise sampled on a lattice that is fixed with respect to the grid used to discretize Eq. (3). This models noise that is correlated over length scales comparable to the coherence length.

First, recall the formation of a deterministic vortex lattice. Vortices nucleate on the sides of the sample and move toward the center. The vortex lattice then rotates into an Abrikosov lattice as seen in Fig. 1, which is for a homogeneous,

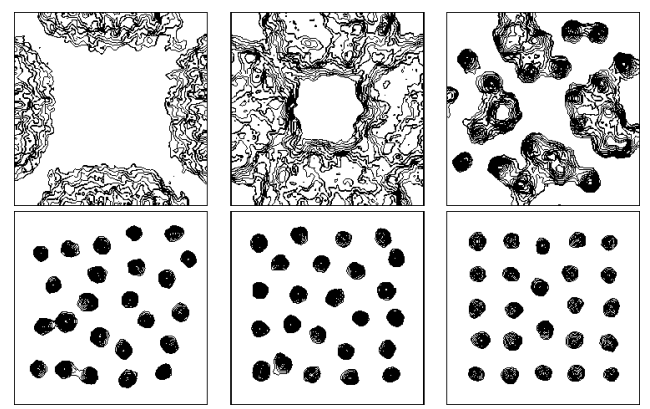

FIG. 2. Time evolution of $|\psi|$ with additive noise of variance 2 in a $20 \xi \times 20 \xi$ sample. The bottom right figure corresponds to a snapshot of the quasi-steady-state configuration.

isotropic, two-dimensional superconducting material of size $20 \xi \times 20 \xi$ in a perpendicular magnetic field of magnitude $0.5 \kappa$ (note that all variables have been appropriately nondimensionalized). The numerical computations are based on a code developed for the steady-state and time-dependent G-L models and several of their variants; see Refs. 2 and 15-18. In the figures, contour lines for the magnitude of the order parameter $\psi$ are plotted; for clarity, only those corresponding to $|\psi| \leqslant 0.5$ are shown. We refer to the isolated regions with $|\psi| \leqslant 0.5$ near the center of vortices as the core regions of the vortices and the diameters of these regions are referred to as the core sizes that are related to the coherence length of the material sample.

\section{RESULTS FOR ADDITIVE NOISE}

Development of a quasi-steady-state vortex lattice. When a given noise with variance 2 is added to the same material sample as that considered in Fig. 1, the lattice no longer rotates into a steady state lattice. Instead, the vortices vibrate in the sample until a lattice similar to the lattice in Fig. 1 is formed; see Fig. 2. A lattice is recognizable in this sample, but a true steady state as seen in Fig. 1 is not achieved since the shapes of the vortices are distorted and the vortices are constantly in motion. However, with this small variance of 2 , the vortices do try to form a regular, triangular pattern in the sample. We refer to the situation in which a persistent lattice is recognizable, but a true steady state is not achieved as a quasi-steady-state configuration. Note that due to the presence of the additive noise the vortices are no longer circular but that the core sizes are much the same, not only from one vortex to another, but also for the same vortex from one time instant to another.

Since the forcing function $\eta$ is random, different computational runs can result not only in different evolutionary histories, but also in different persistent vortex lattice patterns that appear after marching through time. For example, during the time evolution when the vortices enter the sample, the presence of fluctuations can inhibit some vortices from entering. In three other realizations like the one in Fig. 2, three different quasi-steady-states were observed in the separate computations; one is similar to that in Fig. 2, but the lattice is rotated $90^{\circ}$; the other two lattices have 23 vortices compared to the 24 vortices in the steady state lattice without 

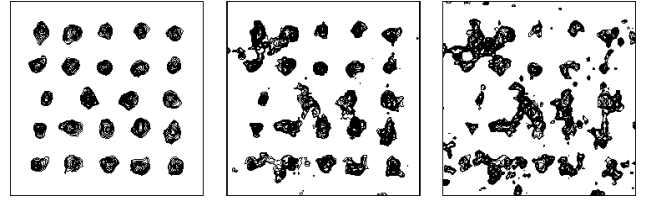

FIG. 3. Snapshots in time of $|\psi|$ for a $20 \xi \times 20 \xi$ sample with additive noise having variances of 3,6 , and 9. All snapshots are taken at the same nondimensionalized time instant.

fluctuations. One vortex is inhibited from entering the material during the nucleation process at the boundary of the sample due to the presence of fluctuations in the model. The two lattices with 23 vortices are similar to each other with a relative $90^{\circ}$ rotation of the lattice structure.

Effect of increasing the variance. In Fig. 3, snapshots taken at the same instant of time for different values of the variance are compared for a $20 \xi \times 20 \xi$ sample. The plots result from a series of calculations with variances equal to 3 , 6 , and 9. For low variances, vortices are recognizable and a vortex lattice is seen. As the variance is increased, the vortices are no longer recognizable and neither is a vortex lattice. In Fig. 4, we give a similar set of plots for a threedimensional computation.

The variance parameter controls the level of thermal fluctuations (or perhaps defects) that are added to the timedependent G-L equations. As the variance increases, the vortices are more distorted and eventually vortices are not recognizable. As discussed earlier, different quasi-steadystates can arise from calculations with the same variance level due to different realizations of the stochastic process that models thermal fluctuations. However, although for the same value of the variance the details of the different realizations may vary (even the number of vortices may be different), the level of distortion of the vortices and the vortex lattices is very similar, e.g., the level of disorder of the individual vortices and of the lattice are similar.

\section{RESULTS FOR MULTIPLICATIVE NOISE}

We first consider noise that is independent in space-time so that the discretized noise is essentially sampled on the same grid as that used for the discretization of the G-L equations. The size of this grid is much smaller than a coherence length. The material sample has dimension $10 \xi \times 10 \xi$ and the

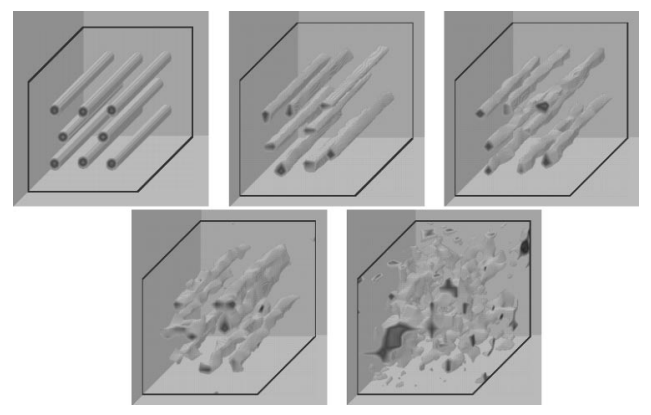

FIG. 4. Snapshots in time of $|\psi|$ for a $15 \xi \times 15 \xi \times 15 \xi$ sample with additive noise having variances of $0,2,5,8$, and 15. All snapshots are taken at the same nondimensionalized time instant.

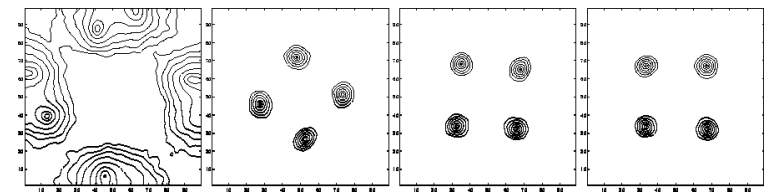

FIG. 5. Level curves of $|\psi|$ for a $10 \xi \times 10 \xi$ sample with spacetime independent multiplicative noise having variance 4 at the nondimensional times 5, 20, 120, 240, and 360 .

applied field is $0.5 \kappa$. Contour plots of the magnitude of the order parameter are given in Figs. 5 and 6 for values of the variances equal to 4 and 8 , respectively. Again, only contour curves corresponding to $|\psi| \leqslant 0.5$ are shown. There are noticeable decreases in core sizes for larger values of the variance while the relative motion of the centers of the vortices is less dramatic than for the additive noise case.

We next consider multiplicative noise in which the noise is sampled on a space-time grid of the scale of a coherence length, i.e., one much coarser than that used to discretize the G-L equations. Some interesting phenomena develop as the less frequent sampling decreases the variations of the coefficients in the noise term and thus gives rise to a change of the effective coherence length over time and space. One can observe that the cores of the vortices undergo constant expansion and shrinkage. For example, consider Fig. 7 in which a $15 \xi \times 15 \xi$ sample with multiplicative noise sampled over a lattice of dimension of a coherence length is considered. In the top row of Fig. 7, we follow the nucleation of vortices at the boundary of the sample and the subsequent migration of the vortices to their quasi-steady-state positions. In the bottom row, we follow the vortices after the quasi-steady-state has been established. We see that not only are the vortex centers moving significantly, but also the sizes of the vortex cores change dramatically in time. Furthermore, at the same time, different vortices can have widely different core sizes.

\section{SUMMARY AND CONCLUSIONS}

Computationally, the effect of thermal fluctuations is evident in the contour plots of the density of superconducting electrons since vortices are in constant motion and are distorted and the vortex lattice is also distorted. With small variances and at large times, the vortices seem to be at an equilibrium where each vortex is on the average at a fixed position in the sample. When the noise is subsequently stopped at this quasi-steady-state, the resulting vortex lattice evolves into a steady state configuration for the sample with respect to the number of vortices present in the material. It can be observed from the numerical simulations that, with no

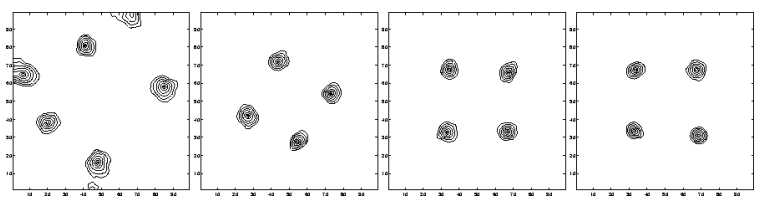

FIG. 6. Level curves of $|\psi|$ for a $10 \xi \times 10 \xi$ sample with spacetime independent multiplicative noise having variance 8 at the nondimensional times 5, 20, 120, 240, and 360 . 


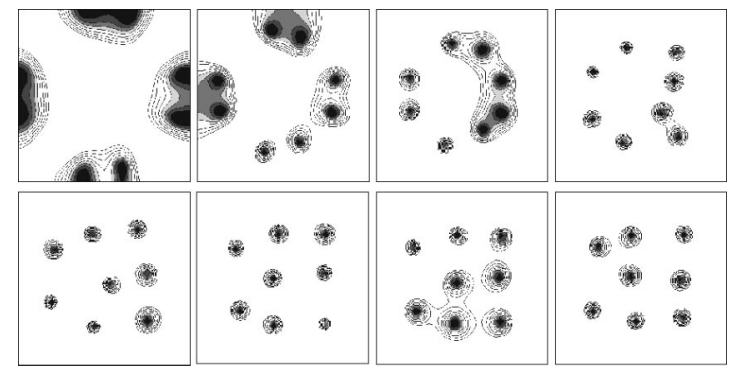

FIG. 7. Level curves of $|\psi|$ at different times for a $15 \xi \times 15 \xi$ sample with multiplicative noise having long range order.

noise present, the free energy monotonically decreases in time, while in the presence of noise the random motion of the vortices results in oscillations in the value of the free energy. In comparison, multiplicative noise produces much larger oscillations, which are perhaps due to the dramatic change of the core sizes.

Although a sample can have several different steady-state lattice structures corresponding to different realizations of the same stochastic process with a given variance, each lattice is a quasi-steady state. Thus, the lattice symmetry and regularity seen in pure materials without fluctuations or defects are still seen with the addition of noise in our models. We also observe that, while additive noise tends to make the positions of vortices vibrate, multiplicative noise tends to alter the core sizes of the vortices, which is even more apparent when discrete lattice random functions are used.

Theoretically, it can also be shown ${ }^{19}$ that as the variance approaches zero the solution of the stochastic high- $\kappa$ timedependent G-L equations approaches the solution of the deterministic high- $\kappa$ time-dependent G-L equations. When the variance decreases to zero, the level of the noise introduced into the stochastic time-dependent G-L equation goes to zero. Intuitively, as the level of the noise goes to zero, the resulting values of $\psi$ at the steady state should approach the values obtained without fluctuations. Therefore, this theoretical result is reflected in the computational studies of quasisteady-state lattices.
*Current address: Lockheed Martin Mission Systems, 700 North Frederick Ave., Gaithersburg, MD 20879.

${ }^{1}$ W. Skocpol and M. Tinkham, Rep. Prog. Phys. 38, 1049 (1975).

${ }^{2}$ J. Chapman, Q. Du, M. Gunzburger, and J. Peterson, Adv. Appl. Math. 5, 193 (1993).

${ }^{3}$ Q. Du and P. Gray, SIAM (Soc. Ind. Appl. Math.) J. Appl. Math. 56, 1060 (1996).

${ }^{4}$ T. Kamppeter, F. Mertens, E. Moro, A. Sánchez, and A. Bishop, Phys. Rev. B 43, 5992 (1991).

${ }^{5}$ M. Tinkham, Introduction to Superconductivity (McGraw-Hill, New York, 1975).

${ }^{6}$ A. Dorsey, M. Huang, and M. Fisher, Phys. Rev. B 45, 523 (1992).

${ }^{7}$ A. Filippov, A. Radievsky, and A. Zelster, Phys. Lett. A 192, 131 (1994).

${ }^{8}$ P. Hohenberg and B. Halperin, Rev. Mod. Phys. 49, 435 (1977).

${ }^{9}$ R. Kato, Y. Enomoto, and S. Maekawa, Phys. Rev. B 47, 5992 (1993).
${ }^{10}$ R. Sasik, L. Bettencourt, and S. Habib, Phys. Rev. B 62, 1238 (2000).

${ }^{11}$ R. Troy and A. Dorsey, Phys. Rev. B 47, 2715 (1993).

${ }^{12}$ S. Ullah and A. Dorsey, Phys. Rev. B 44, 262 (1991).

${ }^{13}$ G. Da Prato and J. Zabczyk, Stochastic Equations in Infinite Dimensions (Cambridge University Press, Cambridge, 1992).

${ }^{14}$ A. Larkin, Zh. Éksp. Teor. Fiz. 58, 1466 (1970) [Sov. Phys. JETP 31, 784 (1970)].

${ }^{15}$ J. Deang, Q. Du, M. Gunzburger, and J. Peterson, Philos. Trans. R. Soc. London, Ser. A 355, 1957 (1997).

${ }^{16}$ Q. Du, M. Gunzburger, and J. Peterson, SIAM Rev. 34, 54 (1992).

${ }^{17}$ Q. Du, M. Gunzburger, and J. Peterson, Phys. Rev. B 46, 9027 (1992).

${ }^{18}$ Q. Du, M. Gunzburger, and J. Peterson, Phys. Rev. B 51, 16194 (1995).

${ }^{19}$ J. Deang, Q. Du, and M. Gunzburger (unpublished). 